\title{
Removal of Impulse Noise Using Iterative Unsymmetrical Trimmed Median Filter
}

\author{
Glincy Mary Jacob \\ Computer Science Department \\ College of Engineering \\ Chengannur, Kerala, India
}

\author{
Tony Sam Thomas \\ Computer Science Department \\ College of Engineering \\ Chengannur, Kerala, India
}

\author{
Rahna K.M \\ Computer Science Department \\ College of Engineering \\ Chengannur, Kerala, India
}

\begin{abstract}
It is universally accepted that Median filter is the best filter known so far. Based on this fact many variants of median filter were developed to improve the performance of the standard median filter. In this paper a new approach for the restoration of gray scale and color images that are highly corrupted by impulse noise is proposed. The algorithm works on low density noise also. The algorithm has three stages firstly, finding the corrupted pixels, secondly de-noising the corrupted pixels; thirdly, minimizing the de-noised image to root image. The article proves that the new approach is guaranteed to converge to root image within a finite number of iterations. The proposed algorithm shows better results than the Standard Median Filter, Recursive Median Filter and Decision based Unsymmetrical Trimmed Median Filter.
\end{abstract}

\section{General Terms}

Spatial Filter, Median Filter, Recursive algorithms, Noise reduction, Impulse Noise Removal.

\section{Keywords}

Decision based algorithm, Median Filter, Recursive median filtering, salt and pepper noise, unsymmetrical trimmed median filter.

\section{INTRODUCTION}

Bit errors in transmission introduce impulse noise in images. Impulse noise is mainly introduced during the signal acquisition stages. For restoration of images contaminated by impulse noise several spatial domain filters have been proposed. Comparative studies show that the standard median filter is the most efficient method to remove impulse noise preserving the edge details [1] [2].

Median filtering is a spatial domain filtering technique that is effective in removing impulsive noise preserving the sharp changes in signal without the extensive blurring and edge destruction that are associated with linear filters. Spectral analysis of the median filter gives no particular remark of the filtering process. Hence, deterministic and statistical properties of median filters are used to explain the filter's action on noisy images. It has been proved that the functional optimization property of the median filtering process can be used to show how median-related filters have the essential properties of smoothing without extensive blurring of the signal [3]. However, the significant disadvantage of Standard Median Filter (MF) is that the filter is effective only at low noise densities. Standard median filter do not retain the edge details of the original image when the noise density exceeds $50 \%$ [4]. Too much signal distortion is introduced and major information such as thin lines and sharp corners are lost.

To overcome these problems, researchers have recently developed several variants of median filters, such as Decision based Algorithm (DBA) [5], Decision Based Unsymmetrical Trimmed Median Filter (DBUTMF) [6], Modified Decision based Unsymmetrical Trimmed Median Filter(MDBUTMF)[7], Max/Median Filter [8], Multistage Median Filter [9], and Recursive Median Filter (RMF) [10]. The above algorithms work effectively only for a small range of noise density [11]. The proposed Iterative Unsymmetrical Trimmed Median Filter (IUTMF) algorithm removes this drawback. It gives a better Peak Signal-to-Noise Ratio (PSNR) and Signal to Noise Ratio (SNR). The algorithm minimizes the Root Mean Square Error (ERMS).

\section{ILLUSTRATION OF IUTMF ALGORITHM}

The proposed filter is divided into three stages; first stage identifies the noisy pixel, second stage de-noises the noisy pixel and third stage reduces the de-noised image to root image [12]. These stages are described in the following subsections.

\subsection{Stage 1: Identifying noisy pixels}

This stage identifies the noisy and noise-free pixels. The process of noise detection can be done as follows:

The two types of impulse noise are salt and pepper noise and random valued noise. Salt and pepper noise comprise of salt noise and pepper noise. Based on [1] and [13] salt noise occurs when the corrupted pixel takes minimum gray level value and pepper noise occurs when the corrupted pixel takes maximum gray level value (i.e. 0 and $\mathrm{L}-1$, where $\mathrm{L}$ is the number of gray levels). Thus, at each pixel location $(i, j)$ of the image $y$, we define the mask $\partial$ by the equation (1).

$$
\partial(i, j)=\left\{\begin{array}{lr}
1 \text { for } y(i, j)=0 \text { or } L-1 \\
0 \quad \text { otherwise }
\end{array}\right.
$$

where 1 represents the noisy pixel and 0 represents the noisefree pixel.

\subsection{Stage 2: De-noising noisy pixels}

The impulse noise is removed as follows:

A two dimensional window of size $\mathrm{N} \times \mathrm{N}$ is selected. For the pixel at center of the window (where $\mathrm{i}=\mathrm{j}$ ), if $\partial(\mathrm{i} . \mathrm{j})$ is 0 , then there are two cases:

\subsubsection{Case 1: All elements of the window are 0 or $L-1$.}

If all the elements of the window are 0 or L-1, then replace the middle element with the mean of the elements of the window. Mathematically,

$$
\begin{gathered}
\text { If } \sum_{i=1}^{N} \sum_{j=1}^{N} \partial(i, j)=N \times N \text { then, at } i=j, \\
y(i, j)=\frac{\sum_{i=1}^{N} \sum_{j=1}^{N} y(i, j)}{N \times N}
\end{gathered}
$$




\subsubsection{Case 2: Not all elements of the window are}

\section{0 or $L-1$.}

If not all elements of the window are 0 or L-1, then replace the middle element with the median of the elements after eliminating 0's and L-1's. Mathematically,

$$
\text { If } \sum_{i=1}^{N} \sum_{j=1}^{N} \partial(i, j)<N \times N \text { then, }
$$

a) Eliminate 0's and L-1's elements of the window.

b) Sort the remaining ' $\mathrm{m}$ ' elements.

c) At $i=j$,

$$
y(i, j)=m / 2 \text { th element }
$$

\subsection{Stage 3: Minimization to root image}

The de-noised image can be minimized to the root image by recursively performing the filtering operation. The recursive median filtering is an optimization operation such that the output of the filter is always defined by the minimum of a cost function of the output state of the filter [10].

The process of repeated applications of recursive filtering can be expressed as in equation (4).

$$
y(n)=\sum_{j=1}^{M-1} y_{j}(n)
$$

where, $y_{j}(n)=$ outputs of previous filtering.

Figure 1 shows the flowchart of the implementation of the proposed method assuming that the gray level is in the range $[0, \mathrm{~L}-1]$.

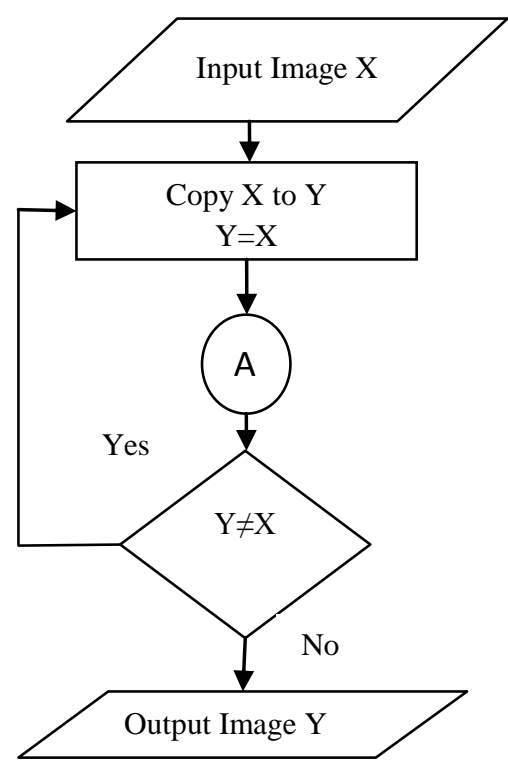

Fig 1(a): Flowchart for IUTMF

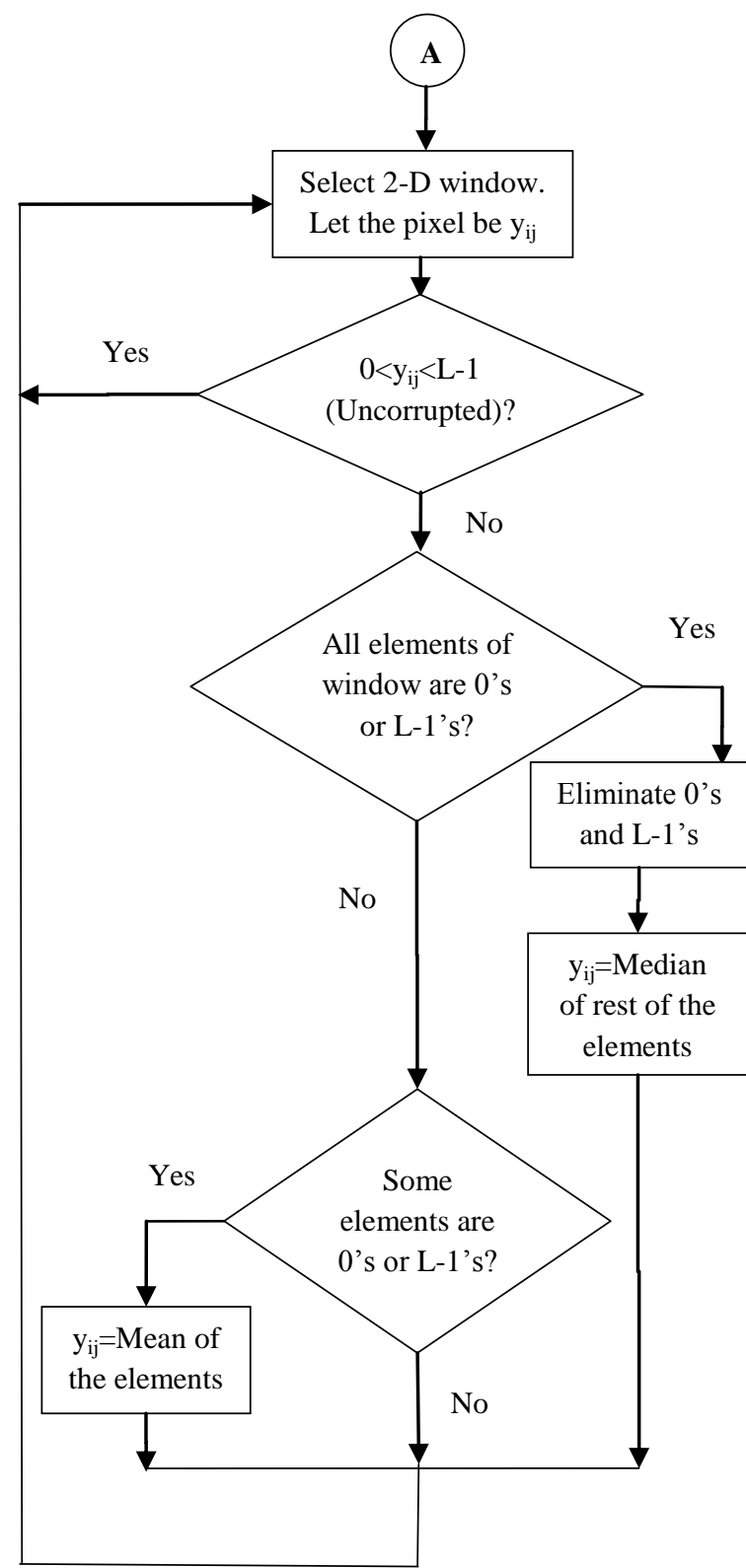

Fig 1(b): Recursive section of the flowchart for IUTMF

\section{PROPOSED ALGORITHM}

The proposed algorithm of Iterative Unsymmetrical Trimmed Median Filter (IUTMF) is elucidated as follows:

Algorithm

Input : Image $\mathrm{x}$

Output : Image y

1. Copy $\mathrm{x}$ to $\mathrm{y}, \mathrm{y}=\mathrm{x}$

2. Select 2-D window of size $\mathrm{N} \times \mathrm{N}$. Let the pixel be $\mathrm{y}_{\mathrm{ij}}$.

3. If $0<y_{i j}<L-1$ then $y_{i j}$ is noise-free pixel and is left unchanged.

4. If $y_{i j}=0$ or $y_{i j}=L-1$ then $y_{i j}$ is noisy pixel, then there are two cases:

Case i): If all the elements of the selected window are 0 's and L-1's, then replace $y_{i j}$ with the mean of the element of window. 
Case ii): If only some elements of the selected window are 0 's and L-1's, then eliminate L-1's or 0 's and find the median value of the remaining elements. Replace $y_{i j}$ with the median value.

5. Repeat steps 2 to 4 until all the pixels in the entire image are processed.

6. While $(y \neq x)$

$$
\begin{array}{ll}
\text { i) } & \mathrm{x}=\mathrm{y} \\
\text { ii) } & \text { Repeat steps } 2 \text { to } 6
\end{array}
$$

\section{SIMULATION RESULTS}

To assess the performance of the new median filtering scheme, the proposed algorithm is tested with different grayscale and color images corrupted by pseudorandom computer generated noise. A $3 \times 3$ window was used in the simulation. The original image (uncorrupted) is shown in Fig. 2 (a). The noisy image is shown in Fig. 2 (b). Fig. 2 (c) shows the filtered image by using Median Filter. Fig 1(d) shows the filtered image using recursive median filter, Fig 2(e) shows that by MDBUTMF. Fig 2(f) shows the filtering by IUTMF.

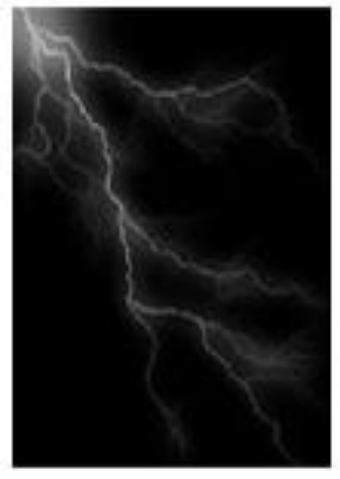

(a)

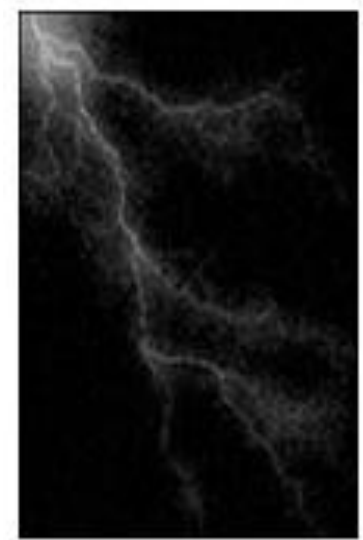

(c)

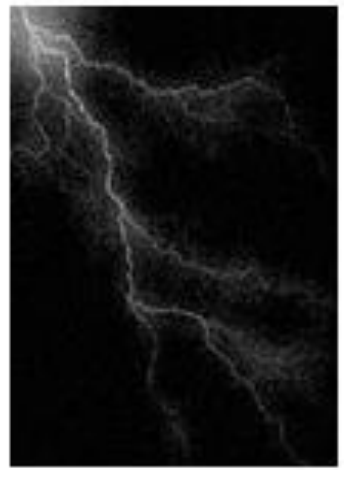

(b)

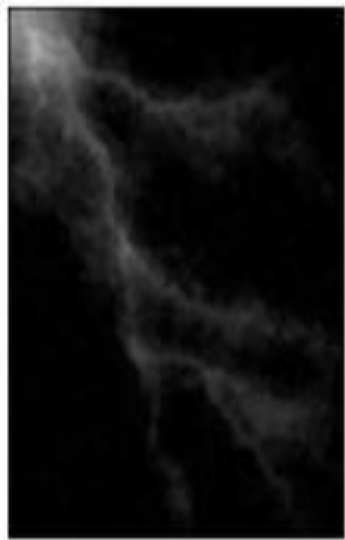

(d)

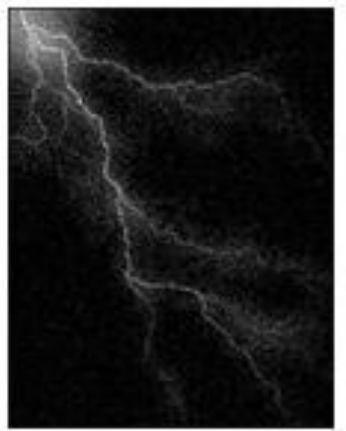

(e)

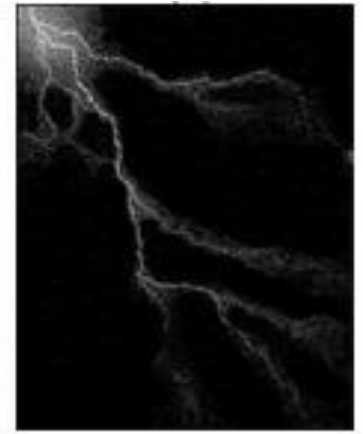

(f)
Fig 2.(a) Original Image; (b) Noisy Image; (c) Filtered image using standard median filter; (d) Filtered image using recursive median filter; (e) Filtered image using MDBUTMF; (f) Filtered image using IUTMF.

\subsection{Experiment 1}

As it can been seen, the differences in visual quality of the filtered images are slight. The filtering performance is quantitatively measured by ERMS, PSNR and SNR as defined in (5), (6) and (7), respectively.

$$
\mathrm{ERMS}=\sqrt{\frac{\sum_{i=1}^{N} \sum_{j=1}^{M}(x(i, j)-\hat{x}(i, j))^{2}}{N \times M}}
$$

where $x(i, j)$ is the original image pixel and $\hat{x}(i, j)$ is the filtered image pixel for an $\mathrm{N} \times \mathrm{M}$ image.

$$
\begin{gathered}
\text { PSNR }=10 \times \log _{10} \frac{255^{2}}{\mathrm{MSE}} \\
\text { where MSE }=\frac{\sum_{\mathrm{i}=1}^{\mathrm{N}} \sum_{\mathrm{j}=1}^{\mathrm{M}}(\mathrm{x}(\mathrm{i}, \mathrm{j})-\widehat{\mathrm{x}}(\mathrm{i}, \mathrm{j}))^{2}}{\mathrm{~N} \times \mathrm{M}} . \\
\qquad \mathrm{SNR}=10 \log _{10} \sum_{\mathrm{i}=1}^{\mathrm{N}} \sum_{\mathrm{j}=1}^{\mathrm{M}} \frac{\hat{\mathrm{x}}(\mathrm{i}, \mathrm{j})^{2}}{[\hat{\mathrm{x}}(\mathrm{i}, \mathrm{j})-\mathrm{x}(\mathrm{i}, \mathrm{j})]^{2}}
\end{gathered}
$$

For a better image the PSNR and SNR ratios must be greater and the ERMS value must be lower. It can be noticed from Table 1, Table 2 and Table 3 that proposed filter gives better result in comparison to other existing techniques. Fig. 3, Fig. 4 and Fig. 5 illustrate the graphical comparison of PSNR, SNR and ERMS performance metric for different filters for gray scale Thunderbolt image.

Table 1: Comparison of PSNR values different algorithms for thunderbolt image at different noise densities.

\begin{tabular}{|c|c|c|c|c|}
\hline $\begin{array}{c}\text { Noise } \\
\text { Density } \\
\text { in \% }\end{array}$ & MF & DBUTM & RMF & IUMF \\
\hline $\mathbf{1 0}$ & -140.986 & -140.105 & -143.718 & -139.97 \\
\hline $\mathbf{2 0}$ & -147.745 & -148.716 & -150.398 & -147.583 \\
\hline $\mathbf{3 0}$ & -152.747 & -152.802 & -155.57 & -152.693 \\
\hline $\mathbf{4 0}$ & -154.892 & -154.439 & -157.887 & -154.373 \\
\hline $\mathbf{5 0}$ & -157.203 & -157.134 & -160.146 & -156.046 \\
\hline $\mathbf{6 0}$ & -159.43 & -160.47 & -163.14 & -158.4 \\
\hline $\mathbf{7 0}$ & -161.08 & -161.8 & -164.86 & -160.14 \\
\hline $\mathbf{8 0}$ & -162.02 & -162.2 & -165.63 & -161.13 \\
\hline $\mathbf{9 0}$ & -163.3 & -162.74 & -167.02 & -161.69 \\
\hline
\end{tabular}


Table 2: Comparison of SNR values different algorithms for thunderbolt image at different noise densities.

\begin{tabular}{|c|c|c|c|c|}
\hline $\begin{array}{c}\text { Noise } \\
\text { Density } \\
\text { in \% }\end{array}$ & MF & DBUTM & RMF & IUMF \\
\hline $\mathbf{1 0}$ & 7.487 & 9.156 & 6.646 & 9.553 \\
\hline $\mathbf{2 0}$ & 3.562 & 5.249 & 2.798 & 6.42 \\
\hline $\mathbf{3 0}$ & 0.966 & 3.222 & -0.202 & 4.402 \\
\hline $\mathbf{4 0}$ & -0.114 & 2.502 & -1.552 & 3.798 \\
\hline $\mathbf{5 0}$ & -0.859 & 2.145 & -2.538 & 3.221 \\
\hline $\mathbf{6 0}$ & -1.747 & 1.902 & -4.964 & 2.894 \\
\hline $\mathbf{7 0}$ & -2.226 & 1.7 & -5.723 & 2.693 \\
\hline $\mathbf{8 0}$ & -2.494 & 1.593 & -6.253 & 2.59 \\
\hline $\mathbf{9 0}$ & -2.614 & 1.433 & -6.759 & 2.429 \\
\hline
\end{tabular}

Table 3: Comparison of ERMS values different algorithms for thunderbolt image at different noise densities.

\begin{tabular}{|c|c|c|c|c|}
\hline $\begin{array}{c}\text { Noise } \\
\text { Density in } \\
\text { \% }\end{array}$ & MF & MDBUTM & RMF & IUTMF \\
\hline $\mathbf{1 0}$ & 58123 & 52514 & 79601 & 50704 \\
\hline $\mathbf{2 0}$ & 126555 & 141516 & 171573 & 119377 \\
\hline $\mathbf{3 0}$ & 225112 & 226533 & 311563 & 213707 \\
\hline $\mathbf{4 0}$ & 288152 & 344333 & 406816 & 241733 \\
\hline $\mathbf{5 0}$ & 375999 & 418510 & 527609 & 314296 \\
\hline $\mathbf{6 0}$ & 486099 & 548057 & 745081 & 443480 \\
\hline $\mathbf{7 0}$ & 587718 & 638972 & 908633 & 533946 \\
\hline $\mathbf{8 0}$ & 654705 & 668802 & 992389 & 563393 \\
\hline $\mathbf{9 0}$ & 759385 & 711726 & 1164250 & 607312 \\
\hline
\end{tabular}

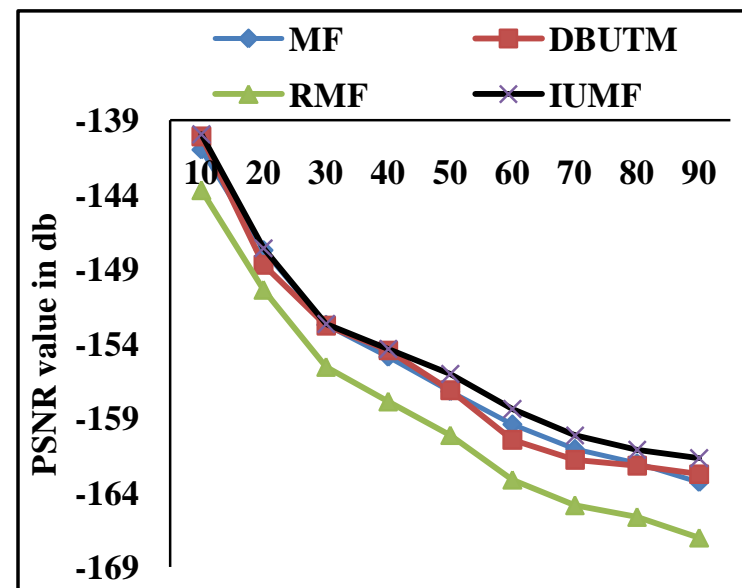

Noise density in \%

Fig 3:PSNR value VS Noise Density in \% for thunderbolt image corresponding to Table 1.

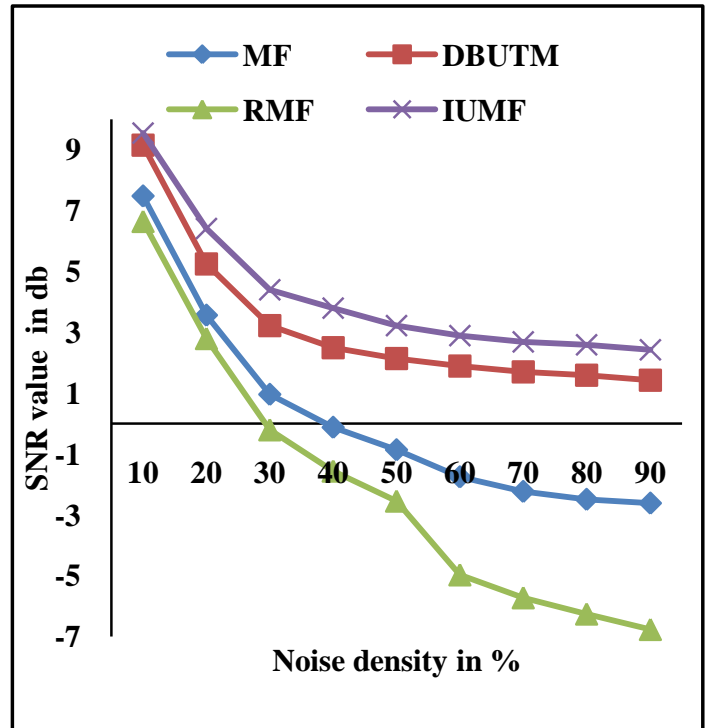

Fig 4: SNR value VS Noise Density in \% for thunderbolt image corresponding to Table 2.



Fig 5: ERMS value VS Noise Density in \% for thunderbolt image corresponding to Table 3.

From Table 1 and Fig 3 it is clear that IUTM gives a better PSNR value. Table 2 and Fig 4 show that IUTM yields a better SNR value. Table 3 and Fig 5 show that the output image obtained by IUTM filtering has less root mean square error. Thus, the quantitative analysis proves that IUTM is a better method than the existing techniques.

Significant visual differences between Recursive Median Filter, Modified Decision Based Unsymmetrical Trimmed Median and Iterative Unsymmetrical Trimmed Median Filter are illustrated in Fig 6. Hence, IUTMF yields an image which is visually devoid of noise and quantitatively preserves information in the original image. 


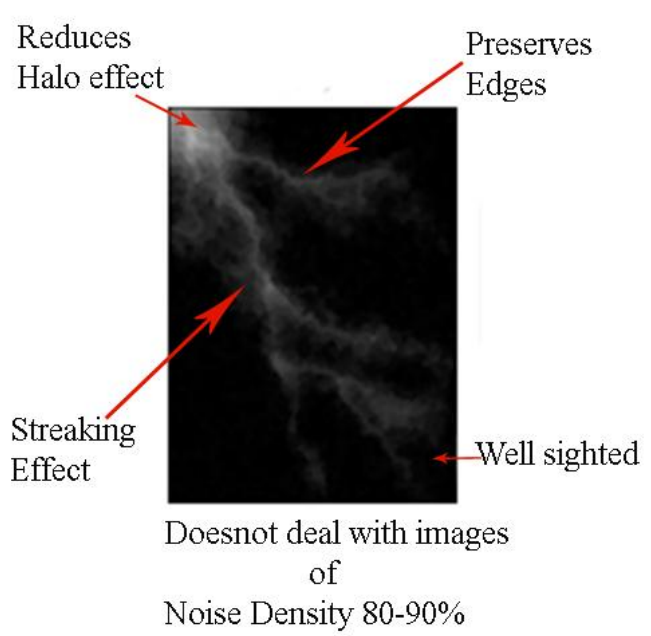

(a)

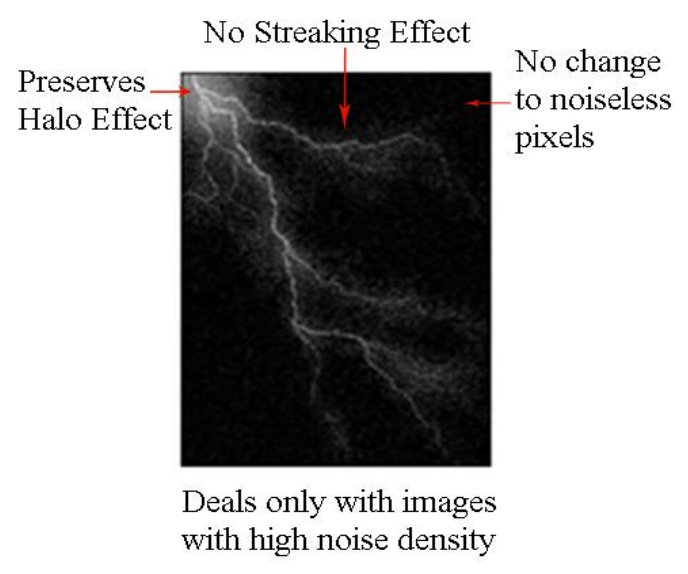

(b)



(c)

Fig 6: Comparison of filtered images by (a) Recursive median filter; (b) MDBUTMF; (c) IUTMF.

\subsection{Experiment 2}

The computational complexity of iterative process is a key factor to determine the cost efficiency of the algorithm. Less complex algorithms are more feasible to implement. An iteration step includes calculating new function values and combining data to calculate the next iterations [14]. Therefore, as the no. of iterations increases, the computational complexity of the algorithm in terms of both time and space also increases.

The proposed algorithm converges the root image in less iteration than the existing median variant filters. Table 4 proves the above statement. Table 4 shows the ERMS value for different iterations. For the thunderbolt image with $10 \%$ noise density, the Recursive Median Filter (RMF) takes 50 iterations to converge the root image while Iterative Unsymmetrical Trimmed Median Filter (IUTMF) takes just 4 iterations.

Table 4: Comparison of ERMS value in different iterations of different filters.

\begin{tabular}{|c|c|c|c|}
\hline ERMS Value & & ilter & \\
\hline $\begin{array}{l}\text { No. of } \\
\text { Iterations }\end{array}$ & DBUTMF & RMF & IUTMF \\
\hline 1 & 52514 & 58123 & 52514 \\
\hline 2 & \multirow{7}{*}{ NA } & 75827 & 51699 \\
\hline 3 & & 79601 & 51095 \\
\hline 4 & & 81819 & 50704 \\
\hline 5 & & 83560 & 50704 \\
\hline 6 & & 84371 & 50704 \\
\hline$\ldots$ & & $\ldots$ & $\ldots$ \\
\hline 50 & & 89662 & 50704 \\
\hline
\end{tabular}

The following observations were found

- There was no change to the noiseless pixels.

- The edges of the filtered image were preserved even at higher noise density.

- The halo effect was reduced without discarding the entire information.

- The image was found to be well-sighted.

- $\quad$ Streaking effect found in Recursive Median Filter is completely reduced.

- $\quad$ The root mean square error is reduced.

- More information about the image is preserved.

- The algorithm converges to the root image in fewer no. of iterations.

\section{CONCLUSIONS}

In this paper, a new algorithm for the elimination of impulse noise is discussed. The goal of the proposed filtering process is to simultaneously satisfy the three tasks of image enhancement: edge preservation, impulsive noise removal, and smoothing of non-impulsive noise. The resultant images look more smoothly filtered and natural. The performance of the algorithm has been tested at low, medium and high noise densities on gray-scale images. Both visual and quantitative 
results are demonstrated. Experimental results prove that the proposed method shows improved filtering of images than Standard Median, Iterative Median and Decision Based Unsymmetrical Trimmed Median Filters. The signal to noise ratio of the image is enhanced. The filtered image has low root mean square error. The algorithm is computationally efficient.

The method is designed for 8 bit images. The method can be enhanced for super resolution images. The proposed method can be improved by extending it to colored images.

\section{REFERENCES}

[1] R. C. Gonzalez, and Woods R.E, "Digital Image Processing". Addison-Wesley, Boston, 2005.

[2] W. K. Pratt, "Median filtering", Image Processing, Institute, University of Southern California, Los Angeles, Technical Report, 1975.

[3] G. Qiu, "Functional optimization properties of median filtering", IEEE Signal Processing Letter., vol. 1, no. 4, pp. 64-65, 1994.

[4] H.Hwang and R.A.Hadded, "Adaptive median filter: New algorithms and results," IEEE Transactions on Image Processing, vol.4, pp499-502.

[5] S. Esakkirajan, T. Veerakumar, Adabala N. Subramanyam and C. H. PremChand, "Removal of High Density Salt and Pepper Noise Through Modified Decision Based Unsymmetric Trimmed Median Filter", IEEE Signal Processing Letters, Vol. 18, NO.5, May 2011.

[6] K.Aiswarya,V.Jayaraj,and D.Ebenezer, "A new and efficient algorithm for the removal of high density salt and pepper noise in image and video," in second International Conference on Computer Modeling and Simulation, 2010, pp.409-413.
[7] K.S.Srinivasan and D.Ebenezer, "A new fast and efficient decision based algorithm for removal of high density impulse noise," IEEE signal processing, Letter, vol.14, no.3, pp.189-192, Mar.2007.

[8] G. R. Arce and M. P. McLoughlin, "Theoretical analysis of max/median filters," IEEE Transaction. Acoustic, Speech, Signal Processing, vol. ASSP-35, pp. 60-69, 1987.

[9] G. R. Arce and R. E. Foster, "Detail-preserving ranked-order based filters for image processing," IEEE Trans. Acoustic, Speech, Signal Processing, vol. ASSP-37, pp. 83-98, 1989.

[10] Guoping Qiu "An Improved Recursive Median Filtering Scheme for Image Processing", IEEE Transactions on Image Processing, Vol. 5, No. 4, April 1996.

[11] Shyam Lal, Sanjeev Kumar and Mahesh Chandra, "Removal of High Density Salt \& Pepper Noise Through Super Mean Filter for Natural Images", IJCSI International Journal of Computer Science Issues, Vol. 9, Issue 3, No 3, May 2012.

[12] Ulrich Eckhardt, "Root Images of Median Filters Semi-topological Approach", 11th International Workshop on Theoretical Foundations of Computer Vision Dagstuhl Castle, Germany, April 7-12, 2002 Revised Papers, pp176-195.

[13] R. H. Chan, C.W. Ho and M. Nikolova, "Salt-andpepper noise removal by median-type noise detectors and detail preserving regularization", IEEE Transactions on Image Processing, vol. 14, no. 10, 2005, pp. 1479-1485.

[14] J. F. Traub," Computational Complexity of Iterative Processes", SI AM J. COMPUT. Vol. 1, No. 2, June, 1972 . 